Musées, Patrimoine et Culture scientifiques et techniques

$190 \mid 2020$

juillet-août 2020

\title{
Vivre au temps du confinement. Retour sur la collecte participative du Mucem
}

Émilie Girard

\section{OpenEdition}

\section{Journals}

Édition électronique

URL : https://journals.openedition.org/ocim/3936

DOI : 10.4000/ocim.3936

ISSN : 2108-646X

Éditeur

OCIM

Édition imprimée

Date de publication : 1 juillet 2020

Pagination : 18-23

ISSN : 0994-1908

Référence électronique

Émilie Girard « Vivre au temps du confinement. Retour sur la collecte participative du Mucem », La Lettre de I'OCIM [En ligne], 190 | 2020, mis en ligne le 01 juillet 2021, consulté le 17 juillet 2021. URL http://journals.openedition.org/ocim/3936 ; DOI : https://doi.org/10.4000/ocim.3936

Ce document a été généré automatiquement le 17 juillet 2021.

Tous droits réservés 


\title{
Vivre au temps du confinement. Retour sur la collecte participative du Mucem
}

\author{
Émilie Girard
}

Une machine à pâtes sera-t-elle parmi les objets du confinement ? Sfogliatrice, machine à pâtes, Italie, 1961.

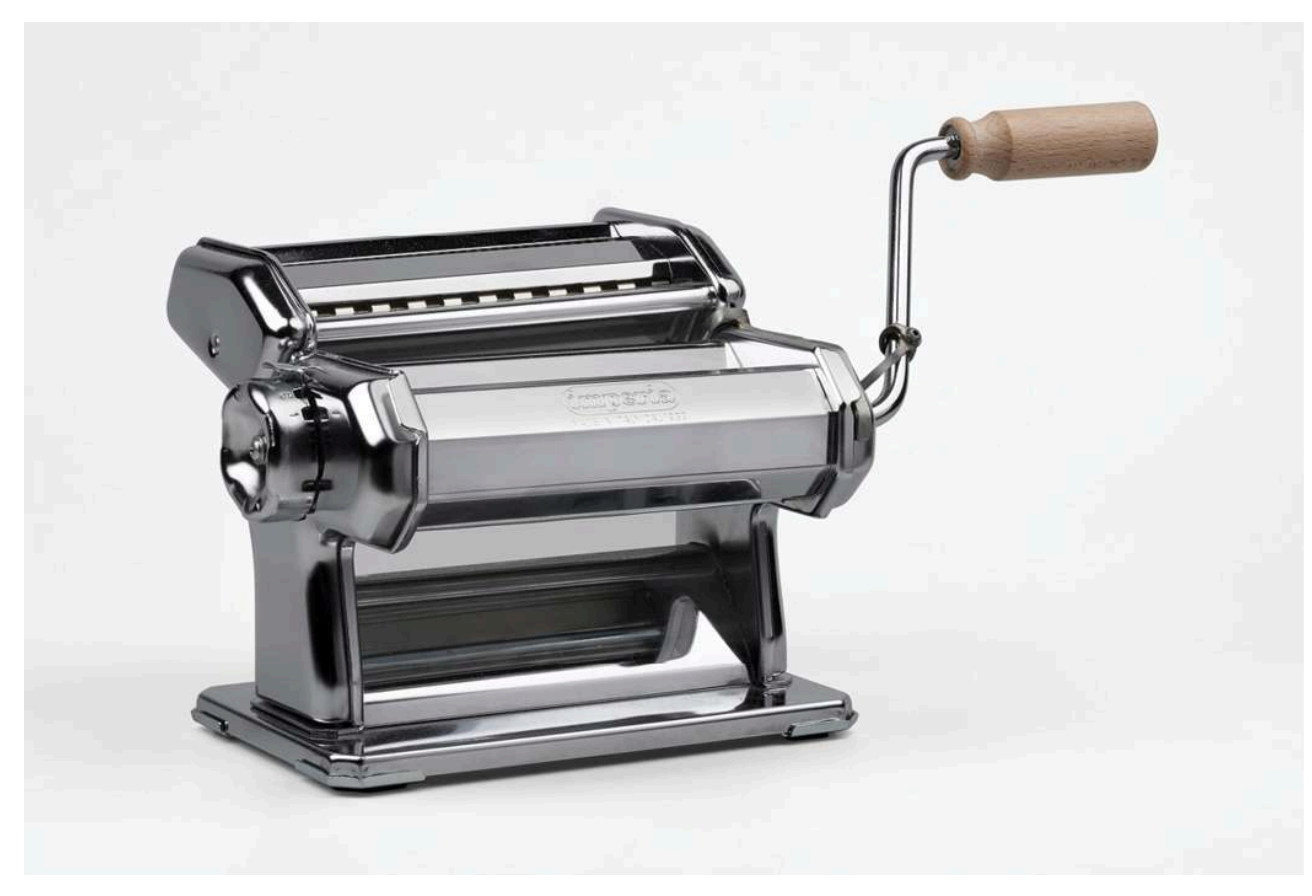

(c) Mucem

Le 20 avril 2020, alors que la France est confinée depuis un mois, le Mucem, musée des Civilisations de l'Europe et de la Méditerranée, lançait sur son site Internet ${ }^{1}$ et sur les réseaux sociaux un appel au don d'objets témoins de la période de confinement. La 
collecte participative Vivre au temps du confinement demandait à ceux qui le souhaitaient de proposer :

2 "les objets ou documents qui symbolisent, incarnent, traduisent votre quotidien confiné. Quels sont selon vous les objets qui parlent de la situation dans laquelle vous vivez, travaillez, passez le temps ou encore enseignez à vos enfants? Quels objets traduisent la manière dont vous organisez vos sorties, vos relations avec les autres, proches ou lointains, chez vous et à l'extérieur, en France ou à l'étranger? Le Mucem est en quête de ces objets qui sont devenus les indispensables de nos vies confinées, attendus ou surprenants, officiels ou bricolés, créateurs de liens ou symboles d'isolement, traduisant les formidables solidarités et soutiens qui se mettent en place ou au contraire les mouvements de rejet et de peur... »

3 Les propositions, attendues jusqu'au 31 mai 2020, devaient comporter une ou plusieurs photographie(s) (dont certaines, si possible, de l'objet dans son environnement d'utilisation ou de fabrication) ainsi qu'un témoignage de quelques lignes expliquant la raison du don et l'importance de l'objet dans le contexte d'alors.

\section{Une collecte parmi d'autres}

4 Avec cet appel, le Mucem s'engageait dans un processus participatif que de nombreuses institutions patrimoniales ont initié. En France, dès le 18 mars, deux jours seulement après le début du confinement, les Archives départementales des Vosges et les Archives municipales de Beaune lançaient simultanément un appel à la collecte. Naissait alors le projet Mémoire de confinement relayé par France Archives et porté par des dizaines de centres d'archives sur tout le territoire.

5 "Le principe consiste à collecter - sous la forme de fichiers PDF ou JPG, mais aussi de vidéos, récits, photographies, dessins - les témoignages de toutes sortes produits par nos concitoyens contraints à rester chez eux pour lutter contre l'épidémie. Chaque contributeur peut faire don du fonds d'archives qu'il constitue à l'issue du confinement. Ces documents, sources d'histoire vivante et donc sources précieuses, peuvent être envoyés par courriel aux Archives, qui consacre cette remise par un formulaire de don ou dépôt, sans condition, incluant la communication immédiate, ainsi que par un formulaire de cession des droits patrimoniaux. ${ }^{2}$ 
Pendant le confinement et en signe de solidarité, le musée de la Civilisation s'est joint au mouvement Ça va bien aller en illuminant son campanile des couleurs de l'arc-en-ciel.

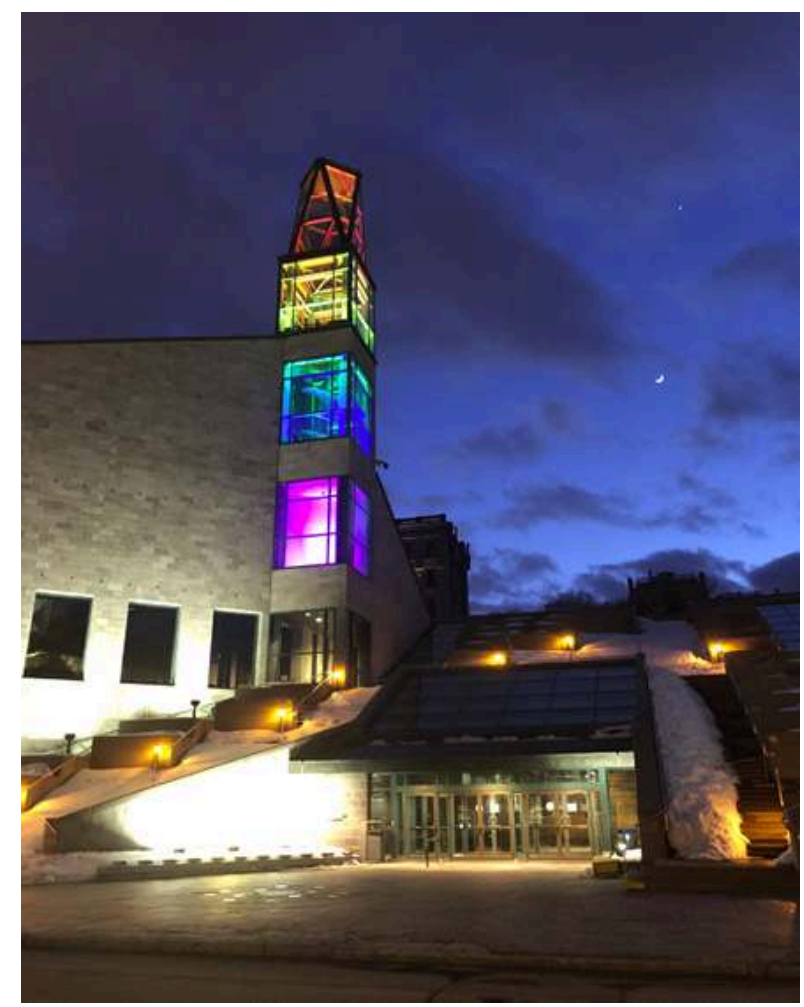

(c) L. Dupuis/Musée de la Civilisation

6 À l'étranger, plusieurs musées se sont également investis dans ce type de collecte. Le musée de la Civilisation de Québec a par exemple invité les citoyens à témoigner de leur expérience à l'aide de dessins d'enfants, de photos, de vidéos ou encore d'écrits. C'est ici moins l'objet qui intéresse le musée que la mise en récits de la période traversée : chaque semaine, les participants sont appelés à répondre à une question. Le procédé choisi permet ainsi de collecter la parole des volontaires en l'inscrivant dans le temps, sujet ô combien important pour l'étude de cette période. À Bruxelles, la Maison de l'Histoire européenne a décidé de s'intéresser aux actions liées à la solidarité en collectant, à travers toute l'Europe, les témoignages matériels et immatériels des manifestations de soutien, d'espoir et de fraternité en ces temps troublés. La New York Historical Society, le Victoria and Albert Museum à Londres, le Deutsches Historisches Museum à Berlin, le Wien Museum, le musée de la Vie wallonne à Liège pour n'en citer que quelques-uns : nombreux sont les musées à rejoindre le monde de la recherche dans un souci de conserver la mémoire d'une période inédite qui bouleverse fondamentalement les habitudes de vie. La tribune rédigée à l'initiative de deux historiennes de l'université de Genève - signée par plus de 100 chercheurs, archivistes, historiens ou sociologues européens, et publiée le 25 avril dans Libération en France ${ }^{3}$, Le Soir en Belgique et Heidi News en Suisse - souligne l'importance de constituer une mémoire collective de la pandémie actuelle, en prenant l'angle de l'histoire sociale.

7 Pour le Mucem, il s'agissait là de la première collecte participative de grande ampleur lancée par le musée, plus habitué des enquêtes-collectes menées sur le terrain par des chercheurs et des conservateurs. Cette année encore, plusieurs de ces campagnes étaient en cours, consacrées à la mémoire orale du Sida, aux savoir-faire et métiers 
romani, aux slogans et graffiti contestataires ou aux retours migratoires en Méditerranée. Mais confinement oblige, il fallait s'adapter et trouver là une méthode différente. L'appel à la participation s'imposait, d'autant qu'il semblait indispensable d'impliquer dans la sélection des objets proposés les premiers concernés par le confinement tout en recueillant leur témoignage.

\section{Quel premier bilan tirer de la collecte du Mucem ?}

8 À l'issue de l'échéance fixée au 31 mai, le Mucem a reçu près de 550 propositions de don émanant de l'ensemble du territoire français, ainsi que quelques propositions étrangères ${ }^{4}$. Toutes les classes d'âge sont représentées parmi les participants à l'appel ; de l'enfant proposant un déguisement de super-héros combattant le virus à la personne âgée offrant une peinture réalisée pendant le confinement. Si à ce stade il est encore difficile de tirer un bilan précis de l'appel, il semble néanmoins que les propositions émanent très majoritairement des catégories sociales les plus favorisées, facteur qu'il conviendra de prendre en compte lors de l'analyse du matériel réuni.

Quelles propositions ont été reçues ? Un premier constat s'impose, celui de la variété des offres. Les objets de prévention, catégorie à laquelle on pouvait s'attendre, sont effectivement présents, mais non majoritaires. Des attestations de sortie et des masques faits maison ont bien sûr été proposés. Certains de ces masques ont fait l'objet d'un souci esthétique particulier : masque en papier plié à la façon des origamis, masque assorti à un jean et des gants ou encore ces masques - coordonnés avec des tabliers - confectionnés dans un tissu imprimé de motifs de croissants et de brioches et utilisés par les vendeuses d'une boulangerie. D'autres marquent une volonté de dédramatisation, comme ceux fabriqués par une mère pour ses enfants, à la façon de déguisements de cow-boy et de soldat. La fabrication en nombre est également évoquée à travers les propositions reçues : une entreprise française de confection de maillots de bain s'étant temporairement reconvertie dans la fabrication de masques offerts au personnel soignant a également proposé un échantillonnage de sa production et quelques prototypes. On trouve également parmi les propositions des visières de protection réalisées à l'imprimante $3 \mathrm{D}$, des objets utilisés pour ouvrir et fermer des portes sans avoir à les toucher ou d'autres accessoires comme une boîte de désinfection pour masques, un thermomètre, ou encore des sur-blouses fabriquées pour être offertes au personnel soignant.

Cette dernière proposition fait le lien avec une autre catégorie, celle des objets témoignant du soutien au monde soignant. Largement médiatisés, les applaudissements de $20 \mathrm{~h}$ et manifestations de solidarité envers le personnel médical ont donné lieu à plusieurs propositions, comme des banderoles fixées aux fenêtres pour remercier médecins, infirmières et aides-soignants, des casseroles, cuillères en bois ou autres ustensiles bricolés servant à faire du bruit à ses fenêtres. 
Les réserves du centre de conservation et de ressources des collections du Mucem pourront accueillir les objets du confinement finalement sélectionnés.

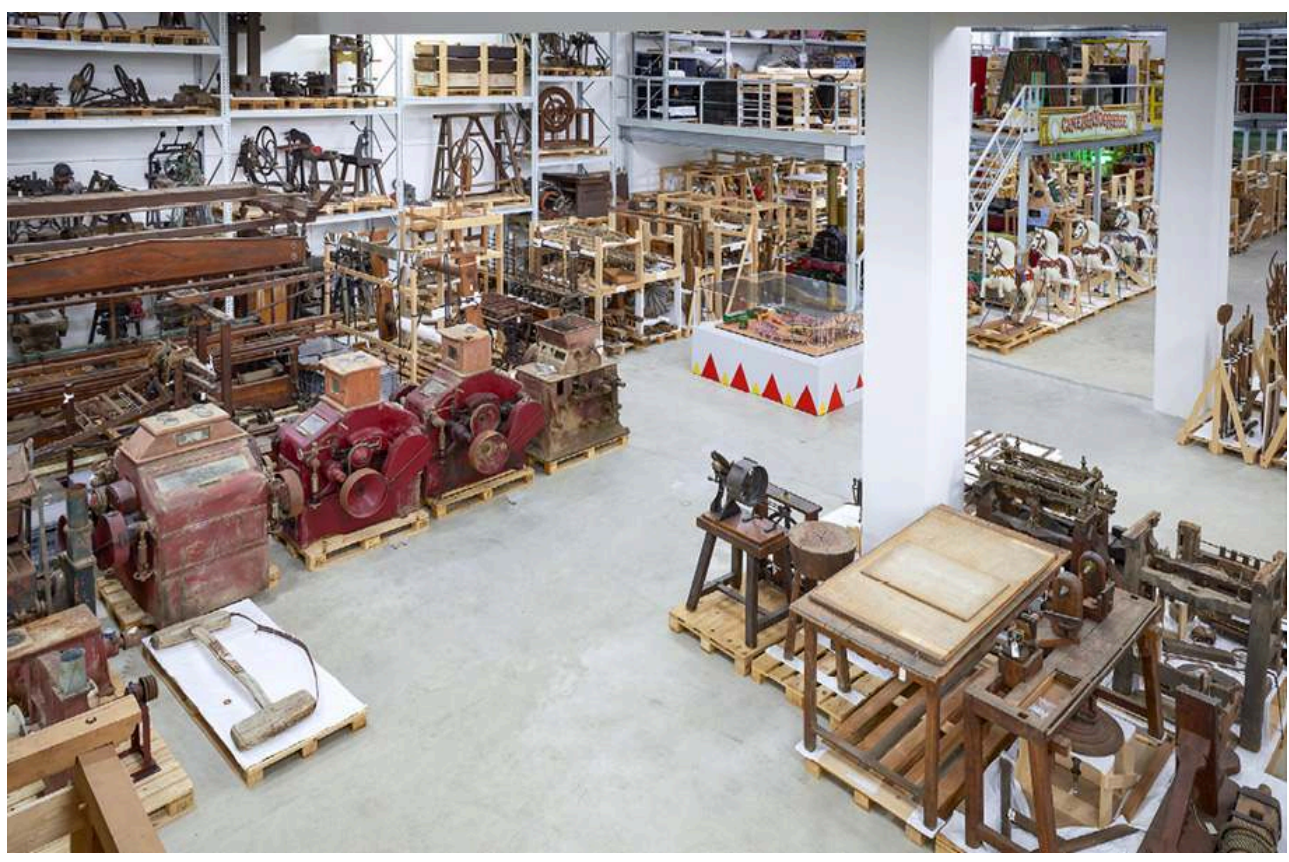

(c) Y. Inchierman/Mucem

11 Les objets témoignant des occupations - habituelles ou originales - faites pour passer le temps, rythmer les journées et permettant de s'investir dans un processus productif ou créatif sont nombreux et représentent sans aucun doute la catégorie la plus nourrie : les réalisations artistiques, souvent inspirées par l'actualité (peintures, dessins, photographies, sculptures, littérature, pratique instrumentale ou chant), sont les plus abondantes (devant les livres, puzzles et jeux divers). Les objets témoins de pratiques sportives sont bien représentés : tapis de yoga ; tableau des calories dépensées jour après jour ; accessoires de sport parfois bricolés, comme une brouette à laquelle un harnachement a été fixé pour être utilisée comme engin à tracter, en y ajoutant le poids de ses enfants.

12 En effet, si l'on en croit certaines propositions reçues, la période de confinement a été propice à l'invention d'objets nécessaires à un nouveau mode de vie. De la tyrolienne reliant deux immeubles se faisant face et permettant de garder un lien avec ses voisins, à la tondeuse à cheveux reliée à l'aspirateur pour éviter la dispersion des cheveux coupés en passant par le fil à désembuer les lunettes... autant d'objets bricolés traduisant une capacité de réaction face à un environnement bouleversé.

La relation au temps a également été profondément modifiée et son appréhension chamboulée se traduit en objets. On retrouve ainsi parmi les propositions faites : des calendriers où les jours de confinement passés sont barrés ; des agendas dans lesquelles les activités réalisées sont inscrites ; des journaux de confinement ; un carnet des menus réalisés quotidiennement ; une compilation de notes manuscrites écrites chaque matin en écoutant les informations à la radio ou une série de "pages de punition " recouvertes chaque jour de confinement de lignes de « je dois rester à la maison ». La durée, le temps qui s'écoule, se matérialisent.

14 Une autre catégorie signifiante peut être définie, celle des objets qui témoignent d'un rapport inédit et modifié à l'intérieur et à l'extérieur. Dans cet ensemble, les chaussures 
sont particulièrement bien représentées, qu'elles soient réservées à un usage domestique (comme les pantoufles), dédiées à la sortie quotidienne autorisée (comme une paire de chaussures de randonnée ou une vieille paire de baskets oubliées réutilisées pour aller courir chaque jour) ou à l'activité professionnelle poursuivie pendant le confinement (chaussures d'un livreur ou d'un infirmier laissées au seuil de la porte tous les soirs). Certaines propositions montrent comment on redécouvre son espace domestique, comme des plans d'appartement dessinés par ses occupants, ou comment on le réinvestit en le réaménageant ou le rangeant. On regarde aussi différemment par sa fenêtre, photographies de vues extérieures à l'appui, on filme des villes endormies ou vivantes malgré la crise, comme Alexandrie, on photographie dans un rayon d'un kilomètre autour de chez soi. La question du passage des frontières est abordée avec la proposition d'un macaron à apposer sur le pare-brise de sa voiture par les travailleurs frontaliers se rendant en Suisse.

Une allégorie du confinement ? Couple sous cloche de verre (France, fin XIX ${ }^{\mathrm{e}}$ siècle-début XX siècle).

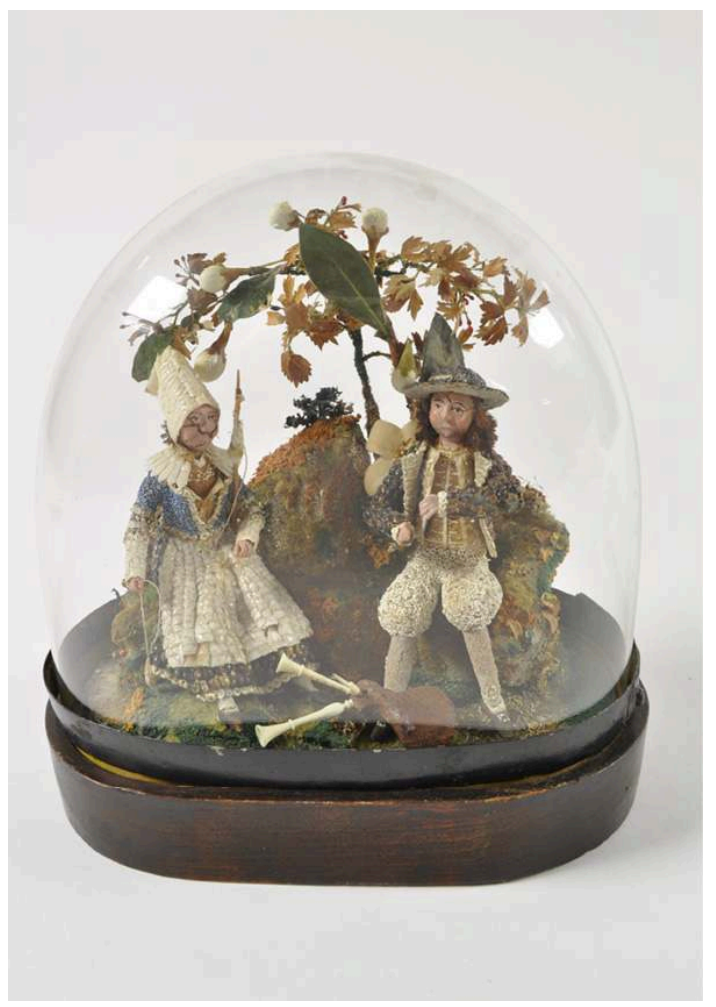

(c) Mucem

D'autres propositions témoignent de la modification profonde et brutale des relations sociales. Les photographies de téléphones, d'ordinateurs ou les copies d'écrans de réunions virtuelles sont nombreuses. On citera l'histoire d'un couple dont le mariage était prévu en avril et dont les proches ont organisé une réunion familiale surprise le jour J via une plateforme de réunion virtuelle et préparé une fausse photographie de noces en réalisant un photomontage réunissant l'ensemble des invités. Une autre personne propose la photographie d'une messe... suivie à la télévision. Si le virtuel a occupé le devant de la scène, la correspondance écrite semble néanmoins avoir fait un retour, comme le montrent quelques propositions d'échanges épistolaires. Face au délitement des relations sociales habituelles, certains participants à l'appel mettent en 
avant l'importance de la compagnie des animaux, du chien que l'on promène aux oiseaux que l'on nourrit sur son balcon, en passant par l'ensemble des animaux vus depuis sa fenêtre et dont on établit une liste.

Des propositions relatives à l'enseignement à la maison ont également été faites : programmes d'enseignement, cahiers de travaux, textes de dictées faites à distance par un grand-parent... Elles émanent de parents, d'élèves mais aussi d'enseignants qui ont sollicité leurs classes ou qui proposent de valoriser leurs projets collectifs.

17 Face à ces objets révélant une forte capacité de réaction et d'adaptation à un contexte inédit, quelques témoignages traduisent les côtés les plus durs de la période traversée : des textes écrits pour les funérailles d'un être disparu évoquent la profonde tristesse de ne pouvoir se réunir en des circonstances difficiles.

18 Au-delà des objets eux-mêmes, il convient de souligner l'importance des textes qui accompagnent les photographies des objets proposés. Ils fournissent bien sûr les informations de contexte nécessaires à la compréhension de la proposition mais sont en outre également très personnels et dénotent souvent l'état d'esprit du donateur. Un dialogue s'est instauré entre les participants à l'appel et le musée, des échanges de mails se sont établis pour remercier ou préciser certains éléments des propositions. Dans un contexte où les relations interpersonnelles étaient profondément modifiées, le musée a indiscutablement joué son rôle social en offrant un espace d'expression particulièrement nécessaire.

\section{Prolonger et nourrir la collecte par la recherche : les prochaines étapes}

19 Toutes les propositions de don ne seront pas retenues. Le travail de sélection a débuté dès la fin de la collecte, en s'appuyant sur des critères de représentativité des différentes catégories, de variété, et de possibilité de conservation à long terme des objets proposés. Les objets sélectionnés seront acheminés jusqu'au musée et prochainement présentés devant les instances décisionnelles en matière d'entrée dans les collections nationales (commission d'acquisition). Mais pas avant une phase d'étude approfondie.

Le Mucem a en effet décidé de prolonger cette collecte en mettant en place un programme de recherche - en partenariat avec universités et chercheurs - qui permettra d'analyser comment la crise sanitaire fait éprouver dans la vie ordinaire (organisation familiale, relations sociales, gestes de tous les jours, travail, circulations) les mutations de nos sociétés à grande échelle (circulations, inégalités, globalisation, environnement, virtualisation): le corpus constitué par la collecte du Mucem représente ainsi une amorce, par le détour de la culture matérielle et des objets, pour étudier ces enjeux. Ils sont des révélateurs de l'impact de la crise sanitaire sur nos modes de vie, nos liens sociaux, nos imaginaires. Le musée s'est donc engagé dans un processus de documentation des objets déjà recueillis (analyse du corpus, documentation complémentaire, entretiens avec les donateurs notamment), d'élargissement du contexte de la collecte (ouverture à l'après confinement, mise en perspective avec des questions plus globales...), de mise en place d'enquêtes de terrain, de mobilisation des partenaires académiques et professionnels (laboratoires de recherche, musées, centres d'archives) impliqués dans des collectes complémentaires 
de celles du Mucem. La mise en relation avec le réseau international des musées ayant lancé des projets similaires a été initiée, avec l'objectif de déboucher, on peut l'espérer, vers une restitution comparée des résultats de cette recherche de grande ampleur. Les journées d'étude portées par la Société Internationale d'Ethnologie et de Folklore et ComCol (comité international d'Icom dédié au développement des collections), prévues en octobre prochain et intitulées An ethnology lab on the workings of Covid-19 on museums ${ }^{5}$ pourraient constituer une première étape de cette mise en commun.

\section{NOTES}

1. Collecte participative, Mucem : https://www.mucem.org/collecte-participative-vivre-autemps-du-confinement

2. Mémoires de confinement, France Archives : https://francearchives.fr/fr/actualite/224765841

3. Piguet M. et Montebello C. Covid-19 : pour une mémoire ordinaire de l'extraordinaire, Libération du 25 avril 2020.

4. Propositions venues d'Espagne, de Belgique, des Émirats arabes Unis, d'Égypte, d'Italie, de Grande-Bretagne et même de Chine et du Mexique.

5. Appel à contribution, The International Society for Ethnology and Folklore : https:// www.siefhome.org/wg/mus/events.shtml

\section{RÉSUMÉS}

Retour sur le processus et les modalités de la collecte participative entreprise par le musée des Civilisations de l'Europe et de la Méditerranée (Mucem) à Marseille pour documenter l'épidémie du Covid-19 et la situation inédite de confinement qui s'est ensuivie.

\section{INDEX}

Mots-clés : dispositif participatif ; écomusée ; musée de société

\section{AUTEUR}

\section{ÉMILIE GIRARD}

Directrice scientifique et des collections au musée des Civilisations de l'Europe et de la Méditerranée.

emilie.girard@mucem.org 